\title{
Lit-only sigma-game on nondegenerate graphs
}

\author{
Hau-wen Huang
}

Received: 31 October 2012 / Accepted: 4 June 2014 / Published online: 25 June 2014 C The Author(s) 2014. This article is published with open access at Springerlink.com

\begin{abstract}
A configuration of the lit-only $\sigma$-game on a graph $\Gamma$ is an assignment of one of two states, on or off, to each vertex of $\Gamma$. Given a configuration, a move of the lit-only $\sigma$-game on $\Gamma$ allows the player to choose an on vertex $s$ of $\Gamma$ and change the states of all neighbors of $s$. Given an integer $k$, the underlying graph $\Gamma$ is said to be $k$-lit if for any configuration, the number of on vertices can be reduced to at most $k$ by a finite sequence of moves. We give a description of the orbits of the lit-only $\sigma$-game on nondegenerate graphs $\Gamma$ which are not line graphs. We show that these graphs $\Gamma$ are 2-lit and provide a linear algebraic criterion for $\Gamma$ to be 1-lit.
\end{abstract}

Keywords Group action · Lit-only $\sigma$-game $\cdot$ Nondegenerate graph

Mathematics Subject Classification Primary 05C57; Secondary 15A63 - 20F55

\section{Introduction}

The notion of the $\sigma$-game on finite graphs $\Gamma$ was first introduced by Sutner $[17,18]$ around 1989. A configuration of the $\sigma$-game on $\Gamma$ is an assignment of one of two states, on or off, to each vertex of $\Gamma$. Given a configuration, a move consists of choosing a vertex of $\Gamma$, followed by changing the states of all of its neighbors. If only on vertices can be chosen in each move, we come to the variation: lit-only $\sigma$-game. Starting from an initial configuration, the goal of the lit-only $\sigma$-game on $\Gamma$ is to minimize the number of $o n$ vertices of $\Gamma$, or to reach an assigned configuration by a finite sequence of moves.

H. Huang $(\varangle)$

Mathematics Division, National Center for Theoretical Sciences, National Tsing-Hua University, Hsinchu 30013, Taiwan, ROC

e-mail: hauwenh@math.cts.nthu.edu.tw 
Given an integer $k$, the underlying graph $\Gamma$ is said to be $k$-lit if for any configuration, the number of $o n$ vertices can be reduced to at most $k$ by a finite sequence of moves. More precisely, we are interested in the orbits of the lit-only $\sigma$-game on $\Gamma$ and the smallest integer $k$, the minimum light number of $\Gamma$ [19], for which $\Gamma$ is $k$-lit. The notion of lit-only $\sigma$-games occurred implicitly in the study of equivalence classes of Vogan diagrams. The Borel-de Siebenthal theorem [2] showed that every Vogan diagram is equivalent to one with a single-painted vertex, which implies that each simply-laced Dynkin diagram is 1-lit. The equivalence classes of Vogan diagrams were described by Chuah and $\mathrm{Hu}$ [7]. A conjecture made by Chang [5,6] that any tree with $k$ leaves is $\lceil k / 2\rceil$-lit was confirmed by Wang and Wu [19], where the name "lit-only $\sigma$-game" was coined.

The lit-only $\sigma$-game on a simple graph $\Gamma$ is simply the natural action of a certain subgroup $H_{\Gamma}$ of the general linear group over $\mathbb{F}_{2}$ [19]. Under the assumption that $\Gamma$ is the line graph of a simple graph $G$, Wu [21] described the orbits of the lit-only $\sigma$-game on $\Gamma$ and gave a characterization for the minimum light number of $\Gamma$. Moreover, if $G$ is a tree of order $n \geq 3$, Wu showed that $H_{\Gamma}$ is isomorphic to the symmetric group on $n$ letters. Weng and the author [13] determined the structure of $H_{\Gamma}$ without any assumption on $G$. The lit-only $\sigma$-game on a simple graph $\Gamma$ can also be considered as a representation $\kappa_{\Gamma}$ of the simply-laced Coxeter group $W_{\Gamma}$ over $\mathbb{F}_{2}$ [12]. The dual representation of $\kappa_{\Gamma}$ preserves a certain symplectic form $B_{\Gamma}$. The two representations are equivalent whenever the form $B_{\Gamma}$ is nondegenerate. From this viewpoint it is natural to partition simple connected graphs into two classes according as $B_{\Gamma}$ is degenerate or nondegenerate.

In this paper, we treat nondegenerate graphs $\Gamma$ which are not line graphs. We show that $H_{\Gamma}$ is isomorphic to an orthogonal group, followed by a description of the orbits of lit-only $\sigma$-game on $\Gamma$ (Theorem 3.1). Moreover, we show that these graphs $\Gamma$ are 2-lit and provide a linear algebraic criterion for $\Gamma$ to be 1-lit (Theorem 3.2). Combining Theorem 3.1, Theorem 3.2, and those in [13,21], the study of the lit-only $\sigma$-game on nondegenerate graphs is quite completed, and the focus for further research is on degenerate graphs.

\section{Preliminaries}

From now on, let $\Gamma=(S, R)$ denote a finite simple connected graph with vertex set $S$ and edge set $R$. Let $\mathbb{F}_{2}$ denote the two-element field $\{0,1\}$. Let $V$ denote an $\mathbb{F}_{2}$-vector space that has a basis $\left\{\alpha_{s} \mid s \in S\right\}$ in one-to-one correspondence with $S$. Let $V^{*}$ denote the dual space of $V$. For each $s \in S$, we define $f_{s} \in V^{*}$ by

$$
f_{s}\left(\alpha_{t}\right)= \begin{cases}1 & \text { if } s=t \\ 0 & \text { else }\end{cases}
$$

for all $t \in S$. The set $\left\{f_{s} \mid s \in S\right\}$ forms a basis of $V^{*}$ and is called the basis of $V^{*}$ dual to $\left\{\alpha_{s} \mid s \in S\right\}$. Each configuration $f$ of the lit-only $\sigma$-game on $\Gamma$ is interpreted as the vector

$$
\sum_{\text {on vertices } s} f_{s} \in V^{*} .
$$


If all vertices of $\Gamma$ are assigned the off state by $f$, we interpret (2) as the zero vector of $V^{*}$. Given $s \in S$ and $f \in V^{*}$ observe that $f\left(\alpha_{s}\right)=1$ (resp. 0 ) if and only if the vertex $s$ is assigned the on (resp. off) state by $f$.

For each $s \in S$ define a linear transformation $\kappa_{s}: V^{*} \rightarrow V^{*}$ by

$$
\kappa_{s} f=f+f\left(\alpha_{s}\right) \sum_{s t \in R} f_{t} \quad \text { for all } \mathrm{f} \in V^{*}
$$

Fix a vertex $s$ of $\Gamma$. Given any $f \in V^{*}$, if the state of $s$ is $o n$, then $\kappa_{s} f$ is obtained from $f$ by changing the states of all neighbors of $s$, and $\kappa_{s} f=f$ otherwise. Therefore, we may view $\kappa_{s}$ as the move of the lit-only $\sigma$-game on $\Gamma$ for which we choose the vertex $s$ and change the states of all neighbors of $s$ if the state of $s$ is on. In particular $\kappa_{s}^{2}=1$. For any vector space $U$, let $\operatorname{GL}(U)$ denote the general linear group of $U$. Then $\kappa_{s} \in \mathrm{GL}\left(V^{*}\right)$ for all $s \in S$. The subgroup $H=H_{\Gamma}$ of $\mathrm{GL}\left(V^{*}\right)$ generated by the $\kappa_{S}$ for all $s \in S$ was first mentioned by Wu [19], which is called the flipping group of $\Gamma$ in [12] and the lit-only group of $\Gamma$ in [21].

The lit-only groups are closely related to the simply-laced Coxeter groups in the following way. Recall that the simply-laced Coxeter group $W=W_{\Gamma}$ associated with $\Gamma=(S, R)$ is the group generated by all elements $s \in S$ subject to the relations

$$
\begin{aligned}
s^{2} & =1, & & \\
(s t)^{2} & =1 & & \text { if } s t \notin R, \\
(s t)^{3} & =1 & & \text { if } s t \in R
\end{aligned}
$$

for all $s, t \in S$. By [12, Theorem 3.2], there exists a unique representation $\kappa=\kappa_{\Gamma}$ : $W \rightarrow \mathrm{GL}\left(V^{*}\right)$ such that $\kappa(s)=\kappa_{s}$ for all $s \in S$. Clearly $\kappa(W)=H$. Given any $f, g \in V^{*}$ observe that $g$ can be obtained from $f$ by a finite sequence of moves of the lit-only $\sigma$-game on $\Gamma$ if and only if there exists $w \in W$ such that $g=\kappa(w) f$. Given an integer $k$, the underlying graph $\Gamma$ is $k$-lit if and only if for each $\kappa(W)$-orbit $O$ on $V^{*}$, there exists a subset $K$ of $S$ with size at most $k$ such that $\sum_{s \in K} f_{s} \in O$.

We now give the definitions of degenerate and nondegenerate graphs. Let $B=B_{\Gamma}$ denote the symplectic form on $V$ defined by

$$
B\left(\alpha_{s}, \alpha_{t}\right)= \begin{cases}1 & \text { if } s t \in R \\ 0 & \text { else }\end{cases}
$$

for all $s, t \in S$ [16]. The radical of $V$ (relative to $B$ ) is the subspace of $V$ consisting of the vectors $\alpha$ that satisfy $B(\alpha, \beta)=0$ for all $\beta \in V$. The form $B$ is said to be degenerate whenever the radical of $V$ is nonzero and nondegenerate otherwise. The graph $\Gamma$ is said to be degenerate whenever the form $B$ is degenerate, and nondegenerate otherwise. The form $B$ induces a linear map $\theta: V \rightarrow V^{*}$ given by

$$
\theta(\alpha) \beta=B(\alpha, \beta) \quad \text { for all } \alpha, \beta \in V
$$


Since the kernel of $\theta$ is the radical of $V$ and the matrix representing $B$ with respect to the basis $\left\{\alpha_{s} \mid s \in S\right\}$ is the adjacency matrix of $\Gamma$ over $\mathbb{F}_{2}$, the following lemma is straightforward.

Lemma 2.1 Let A denote the adjacency matrix of $\Gamma$ over $\mathbb{F}_{2}$. Then the following are equivalent:

(i) $\Gamma$ is a nondegenerate graph.

(ii) $\theta$ is an isomorphism of vector spaces.

(iii) $A$ is invertible.

Recall that given a simple graph $G$, the line graph of $G$ is a simple graph that has a vertex for each edge of $G$, and two of these vertices are adjacent whenever the corresponding edges in $G$ have a common vertex. The purpose of this paper is to investigate the lit-only $\sigma$-game on nondegenerate graphs which are not line graphs. Thus, it is natural to ask how to determine if a nondegenerate graph is a line graph. We will give two characterizations of nondegenerate line graphs as Proposition 2.4 below.

Lemma 2.2 Let $G$ denote a finite simple connected graph of order n. Assume that $\Gamma$ is the line graph of $G$. Then $\theta(V)$ has dimension $n-1$ if $n$ is odd and has dimension $n-2$ if $n$ is even.

Proof Let $U$ denote the vertex space of $G$ over $\mathbb{F}_{2}$. Define a linear map $\mu: V \rightarrow U$ by

$$
\mu\left(\alpha_{s}\right)=u+v \quad \text { for all } s \in S,
$$

where $u$ and $v$ are the two endpoints of $s$ in $G$. Since $G$ is connected, the image of $\mu$ is the subspace of $U$ consisting of these vectors each of which equals the sum of an even number of vertices of $U$. Define a linear map $\lambda: U \rightarrow V^{*}$ by

$$
\lambda(u) \alpha_{s}= \begin{cases}1 & \text { if } u \text { is incident to } s \text { in } G \\ 0 & \text { else }\end{cases}
$$

for all $u \in U$ and for all $s \in S$. There is only one nonzero vector, the sum of all vertices of $G$, in the kernel of $\lambda$. Since $\theta=\lambda \circ \mu$ and by the above comments, the result follows.

A claw is a tree with one internal vertex and three leaves. A simple graph is said to be claw-free if it does not contain a claw as an induced subgraph. A cut-vertex of $\Gamma$ is a vertex of $\Gamma$ whose deletion increases the number of components. A block of $\Gamma$ is a maximal connected subgraph of $\Gamma$ without cut-vertices. A block graph is a simple connected graph in which every block is a complete graph.

Lemma 2.3 [10, Theorem 8.5]. Let $\Gamma$ denote a simple connected graph. Then $\Gamma$ is the line graph of a tree if and only if $\Gamma$ is a claw-free block graph.

The following proposition follows by combining Lemmas 2.1-2.3. 
Proposition 2.4 Let $\Gamma$ denote a simple connected graph. Then the following are equivalent:

(i) $\Gamma$ is a nondegenerate line graph.

(ii) $\Gamma$ is the line graph of an odd-order tree.

(iii) $\Gamma$ is a claw-free block graph of even order.

\section{Main results}

A quadratic form $Q$ on $V$ is a function $Q: V \rightarrow \mathbb{F}_{2}$ satisfying

$$
Q(\alpha+\beta)=Q(\alpha)+Q(\beta)+B(\alpha, \beta) \quad \text { for all } \alpha, \beta \in V \text {. }
$$

Given a quadratic form $Q$ on $V$, the orthogonal group with respect to $Q$ is the subgroup of GL $(V)$ consisting of all $\sigma \in \mathrm{GL}(V)$ such that $Q(\sigma \alpha)=Q(\alpha)$ for all $\alpha \in V$. Given a basis $P$ of $V$ we define $Q_{P}$ to be the unique quadratic form on $V$ with $Q_{P}(\alpha)=1$ for all $\alpha \in P$.

For the rest of this paper, the form $B$ is assumed to be nondegenerate. Moreover, let $Q=Q_{P}$ where $P=\left\{\alpha_{S} \mid s \in S\right\}$ and let $O(V)$ denote the orthogonal group with respect to $Q$. By (6), for any $T \subseteq S$ a combinatorial interpretation of $Q\left(\sum_{s \in T} \alpha_{S}\right)$ is the parity of the number of vertices and edges on the subgraph of $\Gamma$ induced by $T$.

We now can state the main results of this paper, which are Theorem 3.1, Theorem 3.2, and Corollary 3.3.

Theorem 3.1 Assume that $\Gamma$ is a nondegenerate graph, but not a line graph. Then $\kappa(W)$ is isomorphic to $O(V)$. Moreover, the $\kappa(W)$-orbits on $V^{*}$ are

$$
\{0\}, \quad \theta\left(Q^{-1}(0) \backslash\{0\}\right), \quad \theta\left(Q^{-1}(1)\right) .
$$

Under the assumption that $B$ is nondegenerate, the number $|S|=2 m$ is even and there exists a basis $\left\{\beta_{1}, \gamma_{1}, \ldots, \beta_{m}, \gamma_{m}\right\}$ of $V$ such that $B\left(\beta_{i}, \beta_{j}\right)=0, B\left(\gamma_{i}, \gamma_{j}\right)=0$ and

$$
B\left(\beta_{i}, \gamma_{j}\right)= \begin{cases}1 & \text { if } i=j \\ 0 & \text { else }\end{cases}
$$

for all $1 \leq i, j \leq m$. Such a basis $\left\{\beta_{1}, \gamma_{1}, \ldots, \beta_{m}, \gamma_{m}\right\}$ of $V$ is called a symplectic basis of $V$. The Arf invariant of $Q$ is defined to be

$$
\operatorname{Arf}(Q)=\sum_{i=1}^{m} Q\left(\beta_{i}\right) Q\left(\gamma_{i}\right)
$$

which is independent of the choice of the symplectic basis $\left\{\beta_{1}, \gamma_{1}, \ldots, \beta_{m}, \gamma_{m}\right\}$ of $V$ (for example see [1] or [9, Theorem 13.13]). Any two quadratic forms over $\mathbb{F}_{2}$ are equivalent if and only if they have the same Arf invariant and the underlying spaces have the same dimension (for example see [1] or [9, Proposition 13.14]). The order of 
$O(V)$ and the sizes of nontrivial $O(V)$-orbits on $V$ are as follows (cf. [9, Chapter 14]). If $\operatorname{Arf}(Q)=0$ then

$$
\begin{aligned}
|O(V)| & =2^{m^{2}-m+1}\left(2^{m}-1\right)\left(2^{2}-1\right)\left(2^{4}-1\right) \cdots\left(2^{2 m-2}-1\right), \\
\left|Q^{-1}(1)\right| & =2^{2 m-1}-2^{m-1} \\
\left|Q^{-1}(0) \backslash\{0\}\right| & =2^{2 m-1}+2^{m-1}-1 .
\end{aligned}
$$

If $\operatorname{Arf}(Q)=1$ then

$$
\begin{aligned}
|O(V)| & =2^{m^{2}-m+1}\left(2^{m}+1\right)\left(2^{2}-1\right)\left(2^{4}-1\right) \cdots\left(2^{2 m-2}-1\right), \\
\left|Q^{-1}(1)\right| & =2^{2 m-1}+2^{m-1} \\
\left|Q^{-1}(0) \backslash\{0\}\right| & =2^{2 m-1}-2^{m-1}-1 .
\end{aligned}
$$

For each $s \in S$, there exists $\alpha_{s}^{\vee} \in V$ such that

$$
B\left(\alpha_{s}^{\vee}, \alpha_{t}\right)= \begin{cases}1 & \text { if } s=t \\ 0 & \text { else }\end{cases}
$$

for all $t \in S$. The set $\left\{\alpha_{s}^{\vee} \mid s \in S\right\}$ forms a basis of $V$ and is called the basis of $V$ dual to $\left\{\alpha_{s} \mid s \in S\right\}$ (with respect to $B$ ).

Theorem 3.2 Assume that $\Gamma=(S, R)$ is a nondegenerate graph, but not a line graph. Then $\Gamma$ is 2-lit. Moreover, the following are equivalent:

(i) $\Gamma$ is 1-lit.

(ii) The restriction of $Q$ to $\left\{\alpha_{s}^{\vee} \mid s \in S\right\}$ is surjective.

When the nondegenerate graph $\Gamma$ is bipartite, Theorem 3.2 can be improved as follows.

Corollary 3.3 Assume that $\Gamma$ is a nondegenerate bipartite graph. Then $\Gamma$ is 2-lit. Moreover, the following are equivalent:

(i) $\Gamma$ is 1-lit

(ii) $\Gamma$ contains a vertex with even degree or $\Gamma$ is a single edge.

As consequences of Corollary 3.3, we obtain two families of 1-lit graphs as follows.

- A tree is nondegenerate if and only if it has a perfect matching. By [11, Lemma 2.4], a tree with a perfect matching satisfies Corollary 3.3(ii) and is therefore 1-lit (cf. [14, Theorem 1.1]). This result gives a partial affirmative answer for [20, Conjecture 7].

- For any two positive integers $m$ and $n$, the $m \times n$ grid is nondegenerate if and only if $m+1$ and $n+1$ are coprime [18]. By Corollary 3.3 any such $m \times n$ grid is 1-lit. This result partially improves [8, Theorem 26]. 
The following example shows that Corollary 3.3 is no longer true if the assumption of $\Gamma$ is the same as that of Theorem 3.2. Consider the graph $\Gamma=(S, R)$ as below.

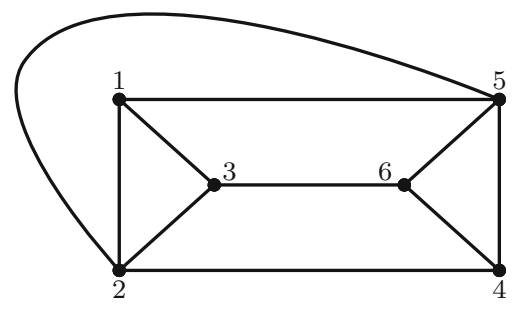

The graph $\Gamma=(S, R)$ is nondegenerate and not a block graph. Therefore $\Gamma$ is not a line graph by Proposition 2.4. The basis $\left\{\alpha_{1}^{\vee}, \alpha_{2}^{\vee}, \ldots, \alpha_{6}^{\vee}\right\}$ of $V$ dual to $\left\{\alpha_{1}, \alpha_{2}, \ldots, \alpha_{6}\right\}$ can be expressed as follows.

$$
\begin{array}{ll}
\alpha_{1}^{\vee}=\alpha_{2}+\alpha_{6}, & \alpha_{4}^{\vee}=\alpha_{3}+\alpha_{5}, \\
\alpha_{2}^{\vee}=\alpha_{1}+\alpha_{3}+\alpha_{5}+\alpha_{6}, & \alpha_{5}^{\vee}=\alpha_{2}+\alpha_{3}+\alpha_{4}+\alpha_{6}, \\
\alpha_{3}^{\vee}=\alpha_{2}+\alpha_{4}+\alpha_{5}, & \alpha_{6}^{\vee}=\alpha_{1}+\alpha_{2}+\alpha_{5} .
\end{array}
$$

A direct computation shows that $Q\left(\alpha_{s}^{\vee}\right)=0$ for all $s \in S$. Therefore $\Gamma$ is not 1-lit by Theorem 3.2, but the vertices 2,5 have even degree in $\Gamma$.

\section{Proof of Theorem 3.1}

To prove Theorem 3.1, we consider a family of linear transformations on $V$ defined as follows. For $\alpha \in V$, the transvection on $V$ with direction $\alpha$ is a linear transformation $\tau_{\alpha}: V \rightarrow V$ defined by

$$
\tau_{\alpha} \beta=\beta+B(\beta, \alpha) \alpha \quad \text { for all } \beta \in V
$$

Observe that $\tau_{\alpha}$ preserves the form $B$ and that $\tau_{\alpha} \in \mathrm{GL}(V)$ since $\tau_{\alpha}^{2}=1$.

For a subset $P$ of $V$ define $T v(P)$ to be the subgroup of GL(V) generated by $\tau_{\alpha}$ for $\alpha \in P$, and define $G(P)$ to be the simple graph whose vertex set is $P$ and where $\alpha, \beta$ in $P$ form an edge if and only if $B(\alpha, \beta)=1$. For any two linearly independent sets $P$ and $P^{\prime}$ of $V$, we say that $P^{\prime}$ is elementary t-equivalent to $P$ whenever there exist $\alpha, \beta \in P$ such that $P^{\prime}$ is obtained from $P$ by changing $\beta$ to $\tau_{\alpha} \beta$. The equivalence relation generated by the elementary $t$-equivalence relation is called the t-equivalence relation [3].

Lemma 4.1 [3, Theorem 3.3]. Let $P$ denote a linearly independent set of $V$. Assume that $G(P)$ is a connected graph. Then there exists $P^{\prime}$ in $t$-equivalence class of $P$ for which $G\left(P^{\prime}\right)$ is a tree.

Lemma 4.2 [15, Lemma 3.7]. Let $P$ denote a linearly independent set of $V$. Assume that $G(P)$ is the line graph of a tree. Then, for each $P^{\prime}$ in the t-equivalence class of $P$, the graph $G\left(P^{\prime}\right)$ is the line graph of a tree. 
A basis $P$ of $V$ is said to have orthogonal type [4] if $P$ is $t$-equivalent to some $P^{\prime}$ for which $G\left(P^{\prime}\right)$ is a tree containing the graph

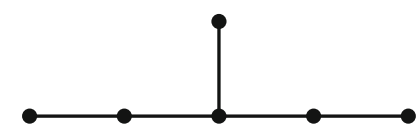

as a subgraph.

Lemma 4.3 Assume that $P$ is a basis of $V$ for which $G(P)$ is a tree, but not a path. Then $P$ is of orthogonal type.

Proof Since $G(P)$ is not a path it contains a vertex $\alpha$ with degree at least three. If any two neighbors of $\alpha$, say $\beta$ and $\gamma$, are leaves of $G(P)$, then $\beta+\gamma$ lies in the radical of $V$, which contradicts that $B$ is nondegenerate. Therefore, at most one neighbor of $\alpha$ is a leaf in $G(P)$ and so $P$ is of orthogonal type.

Lemma 4.4 [4, Section 10]. Let $P$ denote a basis of $V$ which is of orthogonal type. Then $T v(P)$ is the orthogonal group with respect to $Q_{P}$. Moreover, the $T v(P)$-orbits on $V$ are

$$
\{0\}, \quad Q_{P}^{-1}(0) \backslash\{0\}, \quad Q_{P}^{-1}(1) .
$$

Proof of Theorem 3.1. For each $s \in S$, let $\tau_{s}$ denote the transvection on $V$ with direction $\alpha_{s}$. By [16, Section 5], there exists a unique representation $\tau=\tau_{\Gamma}: W \rightarrow \operatorname{GL}(V)$ such that $\tau(s)=\tau_{s}$ for all $s \in S$. For each $w \in W$ the transpose of $\tau\left(w^{-1}\right)$ is equal to $\kappa(w)$. Therefore $\kappa$ is the dual representation of $\tau$. Since $\tau$ preserves the form $B$ we have

$$
\theta \circ \tau(w)=\kappa(w) \circ \theta \quad \text { for all } w \in W
$$

Let $P=\left\{\alpha_{s} \mid s \in S\right\}$. Clearly $T v(P)=\tau(W)$ and $G(P)$ is (isomorphic to) $\Gamma$. By Lemma 4.1 there exists $P^{\prime}$ in $t$-equivalence class of $P$ for which $G\left(P^{\prime}\right)$ is a tree. Since $G(P)$ is not a line graph, the tree $G\left(P^{\prime}\right)$ is not a path by Lemma 4.2. By Lemma 4.3 the basis $P^{\prime}$ of $V$, as well as $P$, is of orthogonal type. By Lemma 4.4, the group $\tau(W)=O(V)$ and the $\tau(W)$-orbits on $V$ are $\{0\}, Q^{-1}(0) \backslash\{0\}$, and $Q^{-1}(1)$. Applying (8) and since $\theta$ is an isomorphism by Lemma 2.1, the result follows.

\section{Proof of Theorem 3.2 and Corollary 3.3}

Recall the basis $\left\{\alpha_{s}^{\vee} \mid s \in S\right\}$ of $V$ from (7). To prove Theorem 3.2 and Corollary 3.3, we introduce a simple graph which includes the information of the values $B\left(\alpha_{s}^{\vee}, \alpha_{t}^{\vee}\right)$ for all $s, t \in S$ as follows.

Define $R^{\vee}$ to be the set consisting of all two-element subsets $\{s, t\}$ of $S$ with $B\left(\alpha_{s}^{\vee}, \alpha_{t}^{\vee}\right)=1$. Define $\Gamma^{\vee}$ to be the simple graph with vertex set $S$ and edge set $R^{\vee}$. We will refer to $\Gamma^{\vee}$ as the dual graph of $\Gamma$. Note that the notion of dual graphs defined above is different from the usual ones in graph theory. The following lemma suggests why the graph $\Gamma^{\vee}$ is of interest. 
Lemma 5.1 For each $s \in S$ we have $\theta\left(\alpha_{s}^{\vee}\right)=f_{s}$.

Proof Let $s, t \in S$ be given. Using (5) and (7), we have $\theta\left(\alpha_{s}^{\vee}\right) \alpha_{t}=1$ whenever $s=t$ and otherwise $\theta\left(\alpha_{s}^{\vee}\right) \alpha_{t}=0$. Comparing this with (1) the result follows.

Lemma 5.2 For each $s \in S$ we have

$$
\alpha_{s}=\sum_{s t \in R} \alpha_{t}^{\vee} .
$$

Proof Fix $s \in S$. By (1), (4), and (5), the vector $\theta\left(\alpha_{S}\right)$ is equal to

$$
\sum_{s t \in R} f_{t}
$$

By Lemma 5.1 the above is equal to

$$
\theta\left(\sum_{s t \in R} \alpha_{t}^{\vee}\right)
$$

Now, by Lemma 2.1(ii) this lemma follows.

Observe that $B_{\Gamma^{\vee}}$ is equivalent to $B$. Therefore $\Gamma^{\vee}$ is a nondegenerate graph. Since $\left\{\alpha_{s} \mid s \in S\right\}$ is the basis of $V$ dual to $\left\{\alpha_{s}^{\vee} \mid s \in S\right\}$, the graph $\Gamma$ is the dual graph of $\Gamma^{\vee}$. By duality Lemma 5.2 implies that

Lemma 5.3 For each $s \in S$ we have

$$
\alpha_{s}^{\vee}=\sum_{s t \in R^{\vee}} \alpha_{t} .
$$

Lemma 5.4 Let $A$ and $A^{\vee}$ denote the adjacency matrices of $\Gamma$ and $\Gamma^{\vee}$ over $\mathbb{F}_{2}$, respectively. Then $A$ and $A^{\vee}$ are inverses of each other.

Proof We show that $A^{\vee} A$ is equal to the identity matrix. Let $s, t \in S$ be given. By the comment below Lemma 5.1 the $(s, t)$-entry of $A$ (resp. $A^{\vee}$ ) is equal to $B\left(\alpha_{s}, \alpha_{t}\right)$ (resp. $B\left(\alpha_{s}^{\vee}, \alpha_{t}^{\vee}\right)$ ). By the definition of $\Gamma^{\vee}$ the $(s, t)$-entry of $A^{\vee} A$ is equal to

$$
B\left(\sum_{s u \in R^{\vee}} \alpha_{u}, \alpha_{t}\right) .
$$

By Lemma 5.3 the vector in the first coordinate of (9) is equal to $\alpha_{s}^{\vee}$. Therefore (9) is equal to 1 if and only if $s=t$ by (7). The result follows.

We are now ready to prove Theorem 3.2.

Proof of Theorem 3.2 In Lemma 5.1 we saw that $\theta\left(\alpha_{s}^{\vee}\right)=f_{s}$ for all $s \in S$. Therefore (i) and (ii) are equivalent by Theorem 3.1. To show that $\Gamma$ is 2-lit, it is now enough to consider the two cases: (a) $Q\left(\alpha_{s}^{\vee}\right)=0$ for all $s \in S$; (b) $Q\left(\alpha_{s}^{\vee}\right)=1$ for all $s \in S$. 
(a) It suffices to show that there exist $s, t \in S$ such that $Q\left(\alpha_{s}^{\vee}+\alpha_{t}^{\vee}\right)=1$. Since the form $B$ is nontrivial there exist $s, t \in S$ such that $B\left(\alpha_{s}^{\vee}, \alpha_{t}^{\vee}\right)=1$. Then the $s$ and $t$ are the desired elements in $S$.

(b) It suffices to show that there exist two distinct $s, t \in S$ such that $Q\left(\alpha_{s}^{\vee}+\alpha_{t}^{\vee}\right)=0$. By our assumption, the graph $\Gamma$ is not a complete graph. Using Lemma 5.4, we deduce that $\Gamma^{\vee}$ is not a complete graph. Therefore there exist two distinct $s, t \in S$ such that $B\left(\alpha_{s}^{\vee}, \alpha_{t}^{\vee}\right)=0$. Such $s$ and $t$ are the desired elements in $S$.

To prove Corollary 3.3, we give a sufficient condition for Theorem 3.2(ii).

Lemma 5.5 Let $\Gamma=(S, R)$ denote a nondegenerate graph. Assume that there exists $s \in S$ with even degree in $\Gamma$ such that

$$
\sum_{\substack{\{u, v\} \subseteq S \\ s u, s v \in R}} B\left(\alpha_{u}^{\vee}, \alpha_{v}^{\vee}\right)=0,
$$

where the sum is over all two-element subsets $\{u, v\}$ of $S$ with $s u, s v \in R$. Then the restriction of $Q$ to $\left\{\alpha_{t}^{\vee} \mid\right.$ st $\left.\in R\right\}$ is surjective.

Proof Apply $Q$ to either side of the equation in Lemma 5.2. Using (6), (10) and $Q\left(\alpha_{S}\right)=1$ to evaluate the resulting equation, we obtain that

$$
\sum_{s t \in R} Q\left(\alpha_{t}^{\vee}\right)=1 .
$$

By (11) there exists a neighbor $u$ of $s$ for which $Q\left(\alpha_{u}^{\vee}\right)=1$. Since $s$ has even degree in $\Gamma$ there exists a neighbor $v$ of $s$ for which $Q\left(\alpha_{v}^{\vee}\right)=0$. The result follows.

Proof of Corollary 3.3. By Proposition 2.4 a nondegenerate bipartite graph $\Gamma$ is a line graph if and only if $\Gamma$ is a path of even order. Since every path is 1-lit, this corollary holds for $\Gamma$ as a line graph. We thus assume that $\Gamma$ is not a line graph. By Theorem 3.2 the graph $\Gamma$ is 2-lit. By Lemma 5.4 we deduce that the graph $\Gamma^{\vee}$ is bipartite with the same bipartition that of $\Gamma$. We use this to show that (i) and (ii) are equivalent.

(ii) $\Rightarrow$ (i): Let $s$ denote a vertex of $\Gamma$ with even degree. Since $\Gamma$ and $\Gamma^{\vee}$ are bipartite graphs with same bipartition, we deduce that $B\left(\alpha_{u}^{\vee}, \alpha_{v}^{\vee}\right)=0$ for any neighbors $u, v$ of $s$ in $\Gamma$. Therefore (10) holds. By Lemma 5.5 the restriction of $Q$ on $\left\{\alpha_{t}^{\vee} \mid s t \in R\right\}$ is onto. Therefore $\Gamma$ is 1-lit by Theorem 3.2.

(i) $\Rightarrow$ (ii): Suppose on the contrary that each vertex of $\Gamma$ has odd degree. Using Lemma 5.4, we deduce that each vertex of $\Gamma^{\vee}$ has odd degree. Let $s$ denote any element of $S$. By Lemma 5.3, $Q\left(\alpha_{s}^{\vee}\right)$ is equal to

$$
Q\left(\sum_{s t \in R^{\vee}} \alpha_{t}\right) .
$$

Since the bipartite graphs $\Gamma$ and $\Gamma^{\vee}$ have the same bipartition, we deduce that $B\left(\alpha_{u}, \alpha_{v}\right)=0$ for any neighbors $u, v$ of $s$ in $\Gamma^{\vee}$. By (6), the summation in (12) 
can be moved out front. Since $Q\left(\alpha_{S}\right)=1$ for all $s \in S$, it follows that (12) is equal to 1, contradicting Theorem 3.2(ii).

Acknowledgments This work was supported by National Center of Theoretical Sciences and Ministry of Science and Technology of Taiwan.

Open Access This article is distributed under the terms of the Creative Commons Attribution License which permits any use, distribution, and reproduction in any medium, provided the original author(s) and the source are credited.

\section{References}

1. Arf, C.: Untersuchungen über quadratische Formen in Körpern der Charakteristik 2 (Teil I). J. Reine Angew. Math. 183, 148-167 (1941)

2. Borel, A., de Siebenthal, J.: Les sous-groupes fermés de rang maximum des groupes de Lie clos. Comment. Math. Helv. 23, 200-221 (1949)

3. Brown, R., Humphries, S.P.: Orbits under symplectic transvections I. Proc. Lond. Math. Soc. 52, 517-531 (1986)

4. Brown, R., Humphries, S.P.: Orbits under symplectic transvections II: the case $K=\mathbb{F}_{2}$. Proc. Lond. Math. Soc. 52, 532-556 (1986)

5. Chang, G.: Graph Painting and Lie Algebra. International and Third Cross-strait Conference on Graph Theory and Combinatorics, Jinhua, China. 26-30 June (2005)

6. Chang, G.: Vogan diagrams and the classification of real simple Lie algebras. A Plenary Talk at the Third Pacific Rim Conference on Mathematics, Shanghai, China. 17-21 August (2005)

7. Chuah, M., Hu, C.: Equivalence classes of Vogan diagrams. J. Algebra 279, 22-37 (2004)

8. Goldwasser, J., Wang, X., Wu, Y.: Minimum light numbers in the $\sigma$-game and lit-only $\sigma$-game on unicyclic and grid graphs. Electron. J. Comb. 18, 214 (2011)

9. Grove, L.C.: Classical Groups and Geometric Algebra. American Mathematical Society, Providence, RI (2002)

10. Harary, F.: Graph Theory. Addison-Wesley, Boston (1969)

11. Hou, Y., Li, J.: Bounds on the largest eigenvalues of trees with a given size of matching. Linear Algebra Appl. 342, 203-217 (2002)

12. Huang, H., Weng, C.: Combinatorial representations of Coxeter groups over a field of two elements. arXiv:0804.2150v2

13. Huang, H., Weng, C.: The edge-flipping group of a graph. Eur. J. Comb. 31, 932-942 (2010)

14. Huang, H.: Two-lit trees for lit-only sigma-game. Linear Algebra Appl. 438, 1057-1066 (2013)

15. Huang, H.: Correction of a theorem on the symmetric group generated by transvections. Q. J. Math. 64, 437-442 (2013)

16. Reeder, M.: Level-two structure of simply-laced Coxeter groups. J. Algebra 285, 29-57 (2005)

17. Sutner, K.: Linear cellular automata and the Garden-of-Eden. Math. Intell. 11, 40-53 (1989)

18. Sutner, K.: The $\sigma$-game and cellular automata. Am. Math. Mon. 97, 24-34 (1990)

19. Wang, X., Wu, Y.: Minimum light number of lit-only $\sigma$-game on a tree. Theor. Comput. Sci. 381, 292-300 (2007)

20. Wang, X., Wu, Y.: Lit-only sigma-game on pseudo-trees. Discret. Appl. Math. 158, 1945-1952 (2010)

21. Wu, Y.: Lit-only sigma game on a line graph. Eur. J. Comb. 30, 84-95 (2009) 